\title{
Single-Machine Scheduling with Piece-Rate Maintenance, Interval Constrained Processing Times and Rejection Penalties
}

\author{
Lei Jin ${ }^{1}$, Xianyu $\mathrm{Yu}^{1,2}$, Zhuojia Dong ${ }^{1}$ \\ 1. College of Economics and Management, Nanjing University of Aeronautics and Astronautics, Nanjing, China \\ 2. Research Centre for Soft Energy Science, Nanjing University of Aeronautics and Astronautics, Nanjing, China \\ Lei_Au@126.com, xyyu@nuaa.edu.cn, 934250613@qq.com
}

\begin{abstract}
This paper investigates single-machine scheduling problems with piece-rate machine maintenance and rejection penalties, where the actual job processing time is required to be in the given time interval otherwise the extra penalty should be paid. Each job should be decided to be scheduled or rejected, and the corresponding penalty should be paid when the job is rejected. This paper aims to find the optimal job sequence, the maintenances frequency and the rejected job set to minimize the sum of both the penalties and the makespan of scheduled jobs, the total number of jobs exceeding the time interval and the total completion time, respectively. Analyzed results show that all the considered problems can be optimally solved in polynomial time. Moreover, numeric experiments are conducted to illustrate the efficiency of the proposed algorithm, and to analyze the positive interacting relationship among rejection punishment, the length of time widow and the scheduling results.
\end{abstract}

Keywords-scheduling; piece-rate maintenance; constrained processing times; rejection penalty

\section{INTRODUCTION}

In some smart manufacturing scenarios, multiple objectives should be considered simultaneously to satisfy the customer demand. For an example, in the engine remanufacturing workshop, the actual processing time of remanufacturing each engine should be in a given time interval to guarantee the quality of polishing and painting process. In order to minimize the operation cost of machines, the total processing times on machines (i.e., the total load of machines) is the key criterion to guide the scheduling decision of remanufacturing workshop. In the following, the maintenance activity is proposed to keep the efficiency of machines, and its cost is a key factor considered by the decision maker of remanufacturing workshop. In addition, the order should be assessed to be accepted or rejected due to the limitation of production resource. This kind of production problems can be modeled as a single machine scheduling problem with interval constrained processing times, machine maintenance and rejection decision.

During the last decade, many studies had triggered into a great interest towards scheduling problems with learning and aging effects. In case of the learning/aging effect, the actual processing time of a job decreases/increases when the job is processed later. Biskup (1999) firstly mentioned the learning effect in scheduling research, while Mosheiov (2001) intro- duced the aging effect into the scheduling research. As follows, many papers (see, e.g., Mosheiov and Sidney (2003), Kuo and Yang (2008), Hsu et al. (2011) and Mosheiov and Oron (2012)) used linear or exponential functions to describe learning or aging effects. For scheduling problems with position-dependent effect, the position-dependent function as specific linear or exponential function was considered by Cheng and Wang (2000), Yang and Yang (2010) and Zhao and Tang (2010). Some other papers (see, e.g., Mosheiov and Sidney (2003), Wang and Wang (2011), Yin et al. (2013) and $\mathrm{Yu}$ and Zhang (2014)) explored to use general position-dependent processing times to analyzed the scheduling problem with learning and aging effects.

The learning and aging effects should be well utilized and controlled in the actual production management. One important method of controlling the aging effect in production scheduling is implementing the machine maintenance activities such that the machine can keep the production efficiency. Many researchers introduced the machine maintenance into the studies of scheduling. Liao and Chen (2003) and Xu et al. (2008) introduced the periodic maintenance activity into the single machine scheduling studies. Yu et al. (2013) and Xue et al. (2014) introduced the piece-rate maintenance into the studies of single machine scheduling. For the recent scheduling studies associated with machine maintenance, the reader can refer to Yang et al. (2012), Mor and Mosheiov (2012), Luo and Liu (2017) and Li et al. (2017). Another method of controlling learning and aging effects is using the time interval to constraint the processing time change caused by learning and aging effects. Yu and Zhang (2014) and Xue et al. (2014) adopted this method to minimize the makespan for the considered single machine scheduling problem.

It is assumed in classic scheduling studies that all the jobs should to be processed. However, to minimize the production fees, some jobs which have lager processing time and bring small profits should be rejected in the actual production processes. Machine scheduling with rejection was first considered by Bartal et al. (1995), they studied a muti-processor scheduling problem to minimize the makespan of the scheduling for accepted jobs plus the total rejection penalty. Engels et al. (1998) considered the scheduling problem of minimizing the sum of the weighted completion times of the accepted jobs plus the sum of the penalties of the rejected jobs. Sengupta (2003) considered the scheduling problem to minimize the 
sum maximum lateness of the scheduled jobs and the total rejection penalty. Zhang et al. (2009) considered single-machine makespan minimization scheduling problems under the job rejection constraint, and they provided a fully polynomial-time algorithms scheme for the case with release dates. Other scheduling studies with rejection can be referred to Cheng and Sun (2009), Zou and Zhang (2014), Zhao and Tang (2010), He et al. (2016) and Gerstl et al. (2017).

In this paper, we simultaneously consider interval constrained actual processing time, piece-rate maintenance and rejection into single machine scheduling problems, and explore to find the optimal polynomial algorithm to minimize the sum of penalties (rejection penalty and the penalty of exceeding time interval) and the scheduled cost (e.g., the total number of jobs exceeding time intervals, the total earliness, the total tardiness).

This paper is organized as follows. We introduce the notation and terminology of this paper in the next section. In Section 3, we propose the main results of this paper. In Section 4 , we conclude this paper.

\section{NOTATION AND TERMINOLOGY}

In this following, the notation and terminology throughout this paper will be introduced.

Table 1. Notations and the corresponding description

\begin{tabular}{|c|c|}
\hline \multicolumn{2}{|c|}{ Notation Description } \\
\hline $\mathrm{n}$ & the number of all jobs; \\
\hline $\mathrm{n}_{\mathrm{S}}$ & the number of jobs in the scheduled job set $S$; \\
\hline $\mathrm{n}_{\overline{\mathrm{S}}}$ & the number of jobs in the rejected job set $\bar{S}$; \\
\hline $\mathrm{p}_{\mathrm{j}}$ & the normal processing time of job j; \\
\hline $\mathrm{k}$ & the number of jobs between two maintenances; \\
\hline $\mathrm{PRM}_{\mathrm{i}}$ & the i th piece-rate maintenance; \\
\hline $\mathrm{m}$ & the maintenance frequency, i.e., $m=\lfloor n / k\rfloor$; \\
\hline $\mathrm{G}_{\mathrm{i}}$ & the ith group of jobs; \\
\hline $\mathrm{n}_{\mathrm{i}}$ & he number of jobs in $G_{i}$; \\
\hline$\sigma$ & the total schedule, i.e., $\sigma=\left[\mathrm{G}_{1}, \mathrm{M}_{1}, \ldots, \mathrm{M}_{\mathrm{m}}, \mathrm{G}_{\mathrm{m}+1}\right]$ \\
\hline$p_{j}^{r}$ & the actual processing time of job $\mathrm{j}$ in the rth position; \\
\hline$a_{j}$ & he lower limit of the constraint interval of job j; \\
\hline$b_{j}$ & the upper limit of the constraint interval of job j; \\
\hline $\mathrm{G}_{\mathrm{i}}^{\mathrm{t}}$ & the total actual processing time of jobs in the ith group; \\
\hline $\mathrm{J}_{[1, \mathrm{r}]}$ & the job scheduled in the rth position of lth group; \\
\hline $\mathrm{p}_{[\mathrm{l}, \mathrm{r}]}$ & the normal processing time of job $\mathrm{J}_{[1, \mathrm{r}]}$ \\
\hline $\mathrm{p}_{[\mathrm{l}, \mathrm{r}]}^{\mathrm{r}}$ & the actual processing time of job $\mathrm{J}_{[1, \mathrm{r}]}$ \\
\hline & the time length of ith maintenance; \\
\hline & the completion time of job j; \\
\hline $\mathrm{C}_{[1, \mathrm{r}]}$ & the completion time of job $\mathrm{J}_{[1, \mathrm{r}]}$; \\
\hline $\mathrm{C}_{\max } \mathrm{t}$ & the makespan of scheduled jobs; \\
\hline$\sum_{j=1}^{n} C_{j} t$ & the total completion time of all jobs; \\
\hline$E_{j} \quad t$ & the earliness time of job j, i.e., $E_{j}=\max \left\{0, a_{j}-p_{j}^{r}\right\}$; \\
\hline & the tardiness time of job j, i.e., $T_{j}=\max \left\{0, p_{j}^{r}-b_{j}\right\}$; \\
\hline$\sum_{\mathrm{i}=1}^{\mathrm{n}} \mathrm{L}_{\mathrm{j}}$ & the total number of jobs exceeding the time interval; \\
\hline$\sum_{\bar{S}} e_{j}$ & the total cost of all rejected jobs. \\
\hline
\end{tabular}

The formulation of the considered problem is proposed as follows. There are $\mathrm{n}$ jobs ready to be processed on a single machine. Preemption is not allowed and the machine is only able to process one job at a time. For job $j$, has a normal processing time $\mathrm{p}_{\mathrm{j}}$. The completion time of job $j$ is denoted by $\mathrm{C}_{\mathrm{j}}$. In a schedule, jobs processed continuously form a group. Thus, the schedule can be denoted as $\sigma=\left[G_{1}, M_{1}, \ldots, M_{m}, G_{m+1}\right]$, where $G_{i}$ is the $i$ th group and $\mathrm{M}_{\mathrm{i}}$ is the $i$ th maintenance. $C_{[l, r]}$ is the completion time of the job scheduled in $r$ th position of the $l$ th group.

If job $\mathrm{j}$ is assigned to the $\mathrm{r}$ th position, we denote the actual job processing time by

$$
p_{j}^{r}=f_{j}\left(p_{j}, r\right),\left(1 \leq j \leq n, 1 \leq r \leq n_{i}\right) .
$$

From equation (1), it can be seen that these functions are also not restricted to be specific and monotonic (either decreasing to reflect learning, or increasing to reflect aging), and different jobs can have different functions.

Based on the assumption of piece-rate maintenance, which is introduced in Yu et al. (2013), a maintenance activity is implemented once every $k$ jobs completed, and a maintenance activity is just finished at time zero. During the maintenance activity the machine is turned off and production is stopped. After maintenance, the machine will be restored to its initial condition. This paper names this type of maintenance as piece-rate maintenance, and it can be denoted as $P R M=k$.

For a given schedule, let $S$ denoted the set of accepted jobs, and let $\bar{S}$ denoted the set of rejected jobs. The problem aims to find the set of $S$ and $\bar{S}$ to minimize the sum of the scheduling criterion and the total rejection penalty. Let $h$ denote the number of rejected jobs, then the number of scheduled jobs is $n-h$, where $0 \leq h \leq n$, And we consider the problem with $\bar{S} \neq 0$ and $S$ have $(n-h)(1 \leq h \leq n)$ accepted jobs. The objective function consists of a scheduling criterion of $h$ accepted jobs and a rejection penalty of $h$ rejected jobs. We assumed the accepted jobs are scheduled in the first $(n-h)$ positions and the rejected jobs are in the remaining $h$ positions. Let $\mathrm{m}$ be the maintenance frequency, if the remainder of $(n-h) / k$ is not equal to zero, then $m=\lfloor(n-h) / k\rfloor$, otherwise $m=\lfloor(n-h) / k\rfloor-1$. It can be obtained that

$$
\begin{aligned}
& \sum_{i=1}^{m+1} n_{i}+h=n, n_{i}=k(i=1,2, \ldots, m) \text { and } n_{m+1}= \\
& n-h-m k
\end{aligned}
$$

Let $G_{i}^{t}$ represent the time length of $i$ th group, and $t_{i}$ be the duration of the maintenance, i.e., $t_{i}=a G_{i}^{t}+b$, where a and $\mathrm{b}$ are two constant numbers, $a \geq 0$. Let $J_{[i, r]}$ be the $r$ th job in the group $G_{i}$, which can be denoted by $G_{i}=$ $\left[J_{[i, 1]}, J_{[i, 2]}, \ldots, J_{\left.[i, n-1], J_{[i, n]}\right]}\right.$. The normal processing time and actual processing time of job $J_{[l, r]}$ are respectively denoted as $p_{[l, r]}$ and $p_{[l, r]}^{r}$, where $p_{[l, r]}^{r}=f_{[l, r]}\left(p_{[l, r]}, r\right)$.

The formulation of the objectives is present as follows. Let $L_{j}$ be the $0-1$ variable if $a_{j} \leq p_{j}^{r} \leq b_{j}$ it is equal to 0 otherwise it is equal to 1 . Then, $\sum_{i=1}^{n-h} L_{j}$ is the total number of all jobs whose actual processing time exceeds the time interval $\left[a_{j}, b_{j}\right]$.

The actual processing time of job $j$ is required in the time interval $\left[a_{j}, b_{j}\right]$ otherwise a penalty should be paid. The earliness time of job $j$ according to the time interval $\left[a_{j}, b_{j}\right]$ is denoted as $E_{j}$ i.e., $E_{j}=\max \left\{0, a_{j}-p_{j}^{r}\right\}$; the tardiness time of job $j$ according to the time interval $\left[a_{j}, b_{j}\right]$ is denoted as $T_{j}$ i.e., 
$T_{j}=\max \left\{0, p_{j}^{r}-b_{j}\right\}$. Note that $\left.J_{[i, r]}\right]$ denotes the $r$ th job in the group $G_{i}$. Then, $L_{[l, r]}$ denotes the $0-1$ variable if $a_{j} \leq$ $p_{j}^{r} \leq b_{j}$ it is equal to 0 otherwise it is equal to 1 . Then, we use $L_{[l, r]}$ to denote the $0-1$ variable showing whether the actual processing time of job $j$ exceeds the time interval $\left[a_{j}, b_{j}\right]$ for the job scheduled in the $r$ th position in the $l$ th group or not, i.e.,

$$
\begin{aligned}
& L_{[l, r]}=g\left(p_{[l, r]}, r\right) \\
& =\left\{\begin{array}{c}
0 \text { if } a_{[l, r]} \leq f\left(p_{[l, r]}, r\right) \leq b_{[l, r]} \\
1 \text { if } f\left(p_{[l, r]}, r\right)<a_{[l, r]} \text { or } f\left(p_{[l, r]}, r\right)>b_{[l, r]}
\end{array}\right.
\end{aligned}
$$

The earliness time of job $J_{[i, r]}$ according to the time interval $\left[a_{j}, b_{j}\right]$ is denoted as

$$
E_{[l, r]}=\max \left(0, a_{j}-f\left(p_{[l, r]}, r\right)\right)
$$

Similarly, the tardiness time of job $J_{[i, r]}$ according to the time interval $\left[a_{j}, b_{j}\right]$ is denoted as

$$
T_{[l, r]}=\max \left(0, f\left(p_{[l, r]}, r\right)-b_{j}\right)
$$

In the manufacturing process, the production fees maybe be associated with the total number of jobs exceeding the time interval, the earliness time and the tardiness time. Thus, we consider the total cost as follows:

$$
\mathrm{TC}=\alpha_{1} \sum_{S} L_{j}+\alpha_{2} \sum_{S} E_{j}+\alpha_{3} \sum_{S} T_{j}+\alpha_{4} \sum_{\bar{s}} e_{j}
$$

Where $\alpha_{1}, \alpha_{2}, \alpha_{3}$ and $\alpha_{4}$ are the total number of jobs exceeding the time interval, the earliness time, the tardiness time and the cost of rejected jobs, respectively. We assume that $\alpha_{i} \geq 0, i=1,2, \ldots, 4$.

The total number of all jobs whose actual processing time exceeds the time interval $\left[a_{j}, b_{j}\right]$ is given by

$$
\begin{aligned}
& \sum_{S} L_{j}=\sum_{l=1}^{m+1} \sum_{r=1}^{n_{i}} L_{[l, r]}=\sum_{l=1}^{m} \sum_{r=1}^{k} g\left(p_{[l, r]}, r\right)+ \\
& \sum_{r=1}^{n_{m+1}} g\left(p_{[m+1, r]}, r\right) .
\end{aligned}
$$

Based on the results in Xue et al. (2014), the total earliness time is

$$
\begin{gathered}
\sum_{S} E_{j}=\sum_{l=1}^{m+1} \sum_{r=1}^{n_{i}} E_{[l, r]}=\sum_{l=1}^{m} \sum_{r=1}^{k} \max \left\{0, a_{j}-\right. \\
\left.f\left(p_{[l, r]}, r\right)\right\}+\sum_{r=1}^{n_{m+1}} \max \left\{0, a_{j}-f\left(p_{[m+1, r]}, r\right)\right\} .
\end{gathered}
$$

The total tardiness is given by

$$
\begin{aligned}
& \sum_{s} T_{j}= \\
& \sum_{l=1}^{m+1} \sum_{r=1}^{n_{i}} T_{[l, r]}=\sum_{l=1}^{m} \sum_{r=1}^{k} \max \left\{0, f\left(p_{[l, r]}, r\right)-b_{j}\right\}+ \\
& \sum_{r=1}^{n_{m+1}} \max \left\{0, f\left(p_{[m+1, r]}, r\right)-b_{j}\right\} \text {. }
\end{aligned}
$$

Note that the actual processing times of jobs are assumed to follow equation (3). For convenience, we denote all the problems under consideration using the three-field notation scheme for scheduling problems introduced by Graham et al. (1979).If the number of jobs between two adjacent maintenances $k$ is pre-given, the total cost minimization scheduling problem with interval constrained processing times and piece-rate maintenance and general processing times can be denoted by $1 \mid \mathrm{re} \mathrm{j}, p_{j}^{r}=f_{j}\left(p_{j}, r\right), I C, P R M=k, t_{i}=a G_{i}+$ $b \mid T C$, otherwise the problem is denoted by $1 \mid \mathrm{re} \mathrm{j}, p_{j}^{r}=$ $f_{j}\left(p_{j}, r\right), I C, P R M, t_{i}=a G_{i}+b \mid T C$.

\section{THE MAIN RESULTS}

Theorem 1 The $1 \mid \mathrm{re} \mathrm{j}, p_{j}^{r}=f_{j}\left(p_{j}, r\right), I C, P R M=k, t_{i}=$ $a G_{i}+b \mid T C$ problem can be optimal solved in $O\left(n^{3}\right)$ time.

Proof. The total cost is given by

$$
\begin{aligned}
& \mathrm{TC}=\alpha_{1} \sum_{S} L_{j}+\alpha_{2} \sum_{S} E_{j}+\alpha_{3} \sum_{S} T_{j}+\alpha_{4} \sum_{\bar{S}} e_{j} \\
& =\alpha_{1}\left[\sum_{l=1}^{m} \sum_{r=1}^{k} g\left(p_{[l, r]}, r\right)+\sum_{r=1}^{n_{m+1}} g\left(p_{[m+1, r]}, r\right)\right]+ \\
& \alpha_{2}\left[\sum_{l=1}^{m} \sum_{r=1}^{k} \max \left\{0, a_{j}-f\left(p_{[l, r]}, r\right)\right\}+\sum_{r=1}^{n_{m+1}} \max \left\{0, a_{j}-\right.\right. \\
& \left.\left.f\left(p_{[m+1, r]}, r\right)\right\}\right]+\alpha_{3}\left[\sum _ { l = 1 } ^ { m } \sum _ { r = 1 } ^ { k } \operatorname { m a x } \left\{0, f\left(p_{[l, r]}-b_{j}, r\right)-\right.\right. \\
& \left.\left.b_{j}\right\}+\sum_{r=1}^{n_{m+1}} \max \left\{0, f\left(p_{[m+1, r]}, r\right)\right\}\right]+\alpha_{4} \sum_{\bar{S}} e_{j} \\
& =\alpha_{1} g\left(p_{[l, r]}, r\right)+\alpha_{2} \max \left\{0, a_{j}-f\left(p_{[l, r]}, r\right)\right\}+ \\
& \alpha_{3} \max \left\{0, f\left(p_{[l, r]}, r\right)\right\}+\alpha_{1} g\left(p_{[m+1, r]}, r\right)+ \\
& \alpha_{2} \max \left\{0, f\left(p_{[m+1, r]}, r\right)-b_{j}\right\}+\alpha_{3} \max \left\{0, f\left(p_{[m+1, r]}, r\right)-\right. \\
& \left.b_{j}\right\}+\alpha_{4} \sum_{\bar{s}} e_{j} \\
& =\sum_{l=1}^{m+1} \sum_{r=1}^{n_{l}} f_{[l, r]}^{*}\left(p_{[l, r]}, r\right)+\alpha_{4} \sum_{\bar{S}} e_{j}
\end{aligned}
$$

Where

$$
\begin{aligned}
& f_{[l, r]}^{*}\left(p_{[l, r]}, r\right) \\
& =\left\{\begin{array}{c}
\alpha_{1} g\left(p_{[l, r]}\right)+\alpha_{2} E_{[l, r]}+\alpha_{3} T_{[l, r]}, l=1,2, \ldots, m \\
\alpha_{1} g\left(p_{[m+1, r]}, r\right)+\alpha_{2} E_{[m+1, r]}+\alpha_{3} T_{[m+1, r]}, l=m+1
\end{array}\right.
\end{aligned}
$$

Based on equation (7), the 1|re j, $p_{j}^{r}=f_{j}\left(p_{j}, r\right), I C, P R M=k, t_{i}=a G_{i}+b \mid T C$ problem can be reformulated as a standard assignment problem, which can be described as follows.

$$
\begin{array}{ll}
\min & \sum_{j=1}^{n} \sum_{l=1}^{m+2} \sum_{r=1}^{n_{i}} w_{i l r} x_{j l r}, \\
\text { s.t. } & \sum_{j=1}^{n} x_{j l r}=1, l=1,2, \ldots, m+2, r=1,2, \ldots, n_{i}, \\
& \sum_{l=1}^{m+2} \sum_{r=1}^{n_{i}} x_{j l r}=1, j=1,2, \ldots, n, \\
& x_{j l r}=0 \text { or } 1, \quad j=1,2, \ldots, n, \quad l \\
& =1,2, \ldots, m+2, \quad r=1,2, \ldots, n_{i},
\end{array}
$$

where

$w_{j l r}=\left\{\begin{array}{cl}f_{[l, r]}^{*}\left(p_{[l, r]}, r\right), & l=1,2, \ldots, m+1, r=1,2, \ldots, n_{i} \\ e_{j}, & l=m+2, r=1,2, \ldots, n_{i}\end{array}\right.$

Hence, the problem 1|re j, $p_{j}^{r}=f_{j}\left(p_{j}, r\right), I C, P R M=$ $k, t_{i}=a G_{i}+b \mid T C$ problem can be optimal solved in $O\left(n^{3}\right)$ time.

Corollary 1. The $1 \mid$ re $\mathrm{j}, p_{j}^{r}=f_{j}\left(p_{j}, r\right), I C, P R M=k, t_{i}=$ $a G_{i}+b \mid T C_{1}=\sum_{S}\left(C_{j}-d_{j}\right)+\sum_{\bar{S}} e_{j}$ problem can be optimal solved in $O\left(n^{3}\right)$ time.

Proof. The total cost is given by

$$
\begin{aligned}
& T C_{1}=\sum_{S} C_{j}-d_{j}+\sum_{\bar{S}} e_{j} \\
& \quad=\left[\sum_{l=1}^{m} \sum_{r=1}^{k}(n-h-(l-1) k-r+1+\right. \\
& (n-h-l k) a) f_{[l, r]}\left(p_{[l, r]}, r\right)+\sum_{r=1}^{n_{m+1}}(n-h-m k-r+
\end{aligned}
$$

1) $\left.f_{[m+1, r]}\left(p_{[m+1]}, r\right)+\left((n-h) m b-k b\left(m^{2}+1\right) / 2\right)\right]-$ $\sum_{S} d_{j}+\sum_{\bar{S}} e_{j}$

$$
=\sum_{l=1}^{m+1} \sum_{r=1}^{n_{l}} f_{[l, r]}^{*}\left(p_{[l, r]}, r\right)+\sum_{\bar{S}} e_{j}
$$


where

$$
\begin{gathered}
f_{[l, r]}^{*}\left(p_{[l, r]}, r\right)= \\
\left\{\begin{array}{c}
(n-h-(l-1) k)-r+1+(n-h-l k) a) f_{[l, r]}\left(p_{[l, r]}, r\right), \\
l=1,2, \ldots, m \\
(n-h-m k-r+1) f_{[m+1, r]}\left(p_{[m+1, r]}, r\right), l=m+1
\end{array}\right.
\end{gathered}
$$

We can see this problem can be reformulated as a standard assignment problem, which can be solved in $O\left(n^{3}\right)$.

Corollary 2. The $1 \mid \mathrm{re} \mathrm{j}, p_{j}^{r}=f_{j}\left(p_{j}, r\right), I C, P R M=k, t_{i}=$ $a G_{i}+b \mid T C_{2}=\alpha_{1} \sum_{i=1}^{n-h}\left(C_{j}-d_{j}\right)+\alpha_{2} \sum_{i=1}^{n} L_{j}+$

$\alpha_{3} \sum_{i=1}^{n} E_{j}+\alpha_{4} \sum_{i=1}^{n} T_{j}+\alpha_{5} \sum_{\bar{s}} e_{j}$ problem can be optimal solved in $O\left(n^{3}\right)$ time.

Proof. The total cost is given by

$$
\begin{aligned}
& \quad T C_{2}=\alpha_{1} \sum_{S} C_{j}-d_{j}+\alpha_{2} \sum_{S} L_{j}+\alpha_{3} \sum_{S} E_{j}+\alpha_{4} \sum_{S} T_{j}+ \\
& \alpha_{5} \sum_{\bar{S}} e_{j} \\
& \quad=\alpha_{1} \sum_{l=1}^{m} \sum_{r=1}^{k}[n-h-(l-1) k-r+1+ \\
& (n-h-l k) a] f_{[l, r]}\left(p_{[l, r]}, r\right)+\alpha_{2} \sum_{l=1}^{m} \sum_{r=1}^{k} g\left(p_{[l, r]}, r\right)+ \\
& \alpha_{3} \sum_{l=1}^{m} \sum_{r=1}^{k} \max \left\{0, a_{j}-f\left(p_{[l, r]}, r\right)\right\}+ \\
& \alpha_{4} \sum_{l=1}^{m} \sum_{r=1}^{k} \max \left\{0, f\left(p_{[l, r]}, r\right)-b_{j}\right\}+\alpha_{1} \sum_{r=1}^{n_{m+1}}(n-h- \\
& m k-r+1) f_{[m+1, r]}\left(p_{[m+1, r]}, r\right)+ \\
& \alpha_{2} \sum_{r=1}^{n_{m+1}} g\left(p_{[m+1, r]}, r\right)+\alpha_{3} \sum_{r=1}^{n_{m+1}} \max \left\{0, a_{j}-\right. \\
& \left.f\left(p_{[m+1, r]}, r\right)\right\}+\alpha_{4} \sum_{r=1}^{n_{m+1}} \max \left\{0, f\left(p_{[m+1, r]}, r\right)-b_{j}\right\}+ \\
& \alpha_{1}\left[\left((n-h) m b-\frac{k b\left(m^{2}+1\right)}{2}\right)-\sum_{S} d_{j}\right]+\alpha_{5} \sum_{\bar{s}} e_{j}
\end{aligned}
$$

Where

$$
\begin{gathered}
f_{[l, r]}^{*}\left(p_{[l, r]}, r\right) \\
\qquad \begin{array}{c}
\alpha_{1} \mu_{0} f_{[l, r]}\left(p_{[l, r]}, r\right)+\alpha_{2} g\left(p_{[l, r]}, r\right)+\alpha_{3} E_{[l, r]}+\alpha_{4} T_{[l, r]}, \\
l=1,2, \ldots, m \\
\alpha_{1} v_{0} f_{[l, r]}\left(p_{[l, r]}, r\right)+\alpha_{2} g\left(p_{[m+1, r]}, r\right) \\
+\alpha_{3} E_{[m+1, r]}+\alpha_{4} T_{[m+1, r]}, l=m+1 \\
\mu_{0}=n-h-(l-1) k-r+1+(n-h-l k) a, \\
l=m+1 \\
v_{0}=n-h-m k-r+1, l=m+1
\end{array}
\end{gathered}
$$

Also, we can see the $1 \mid \mathrm{re} \mathrm{j}, p_{j}^{r}=f_{j}\left(p_{j}, r\right), I C, P R M=$ $k, t_{i}=a G_{i}+b \mid \alpha_{1} \sum_{i=1}^{n-h}\left(C_{j}-d_{j}\right)+\alpha_{2} \sum_{i=1}^{n} L_{j}+$ $\alpha_{3} \sum_{i=1}^{n} E_{j}+\alpha_{4} \sum_{i=1}^{n} T_{j}+\alpha_{5} \sum_{\bar{s}} e_{j}$ problem can be reformulated as a standard assignment problem, which can be solved in $O\left(n^{3}\right)$.

Theorem 2 The $1 \mid \mathrm{re} \mathrm{j}, p_{j}^{r}=f_{j}\left(p_{j}, r\right), I C, P R M=k, t_{i}=$ $a G_{i}+b \mid T C$ problem can be optimal solved in $O\left(n^{4}\right)$ time.

Proof. For a fixed $\mathrm{h}$, the $1 \mid \mathrm{re} \mathrm{j}, p_{j}^{r}=f_{j}\left(p_{j}, r\right), I C, P R M=$ $k, t_{i}=a G_{i}+b \mid T C$ problem can be optimally solved via the assignment problem (8) in $O\left(n^{3}\right)$ time. Note that $h$ has $n$ possible values, we need solve the assignment problem for $n$ times. Therefore, to solve the $1\left|\mathrm{re} \mathrm{j}, p_{j}^{r}=f_{j}\left(p_{j}, r\right), I C, P R M=k, t_{i}=a G_{i}+b\right| T C$ problem, the total computational complexity is $O\left(n^{4}\right)$.

In the following, we propose a polynomial time algorithm to optimally solve the $1 \mid \mathrm{re} \mathrm{j}, p_{j}^{r}=f_{j}\left(p_{j}, r\right), I C, P R M=$ $k, t_{i}=a G_{i}+b \mid T C$ problem, where $\mathrm{h}, \mathrm{k}$ is variable, and needs to be determined.

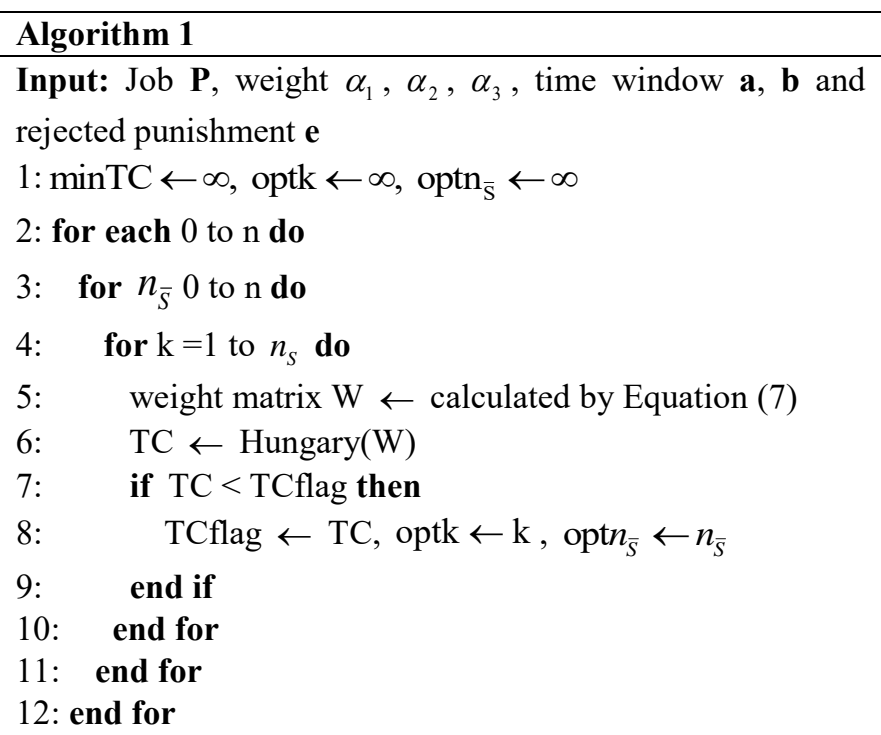

Output: $\operatorname{minTC}$, optk, optn $\overline{\mathrm{S}}_{\overline{\mathrm{S}}}$

Theorem 3 The $1 \mid \mathrm{re} \mathrm{j}, p_{j}^{r}=f_{j}\left(p_{j}, r\right), I C, P R M, t_{i}=a G_{i}+$ $b \mid T C$ problem can be optimal solved in $O\left(n^{5}\right)$ time.

Proof. From Theorem 1, we can see for a given number of jobs in each group $n_{i}, i=1,2, \ldots, m+1, k$ and a given number of rejected jobs $h$, the $1\left|\mathrm{re} \mathrm{j}, p_{j}^{r}=f_{j}\left(p_{j}, r\right), I C, P R M, t_{i}=a G_{i}+b\right| T C \quad$ can be formulated as an assignment problem. The solution of this assignment problem requires an effort of $O\left(n^{3}\right)$ time, Since $n$ different maintenance frequencies and $n$ different rejected jobs need to be evaluated to obtain the optimal schedule. By the Algorithm 1, Steps 3 and 4 are required $n^{2}$ time, so the $1\left|\mathrm{re} \mathrm{j}, p_{j}^{r}=f_{j}\left(p_{j}, r\right), I C, P R M, t_{i}=a G_{i}+b\right| T C \quad$ problem can be optimal solved in $O\left(n^{5}\right)$ time.

\section{NUMERICAL EXAMPLE}

In this section, numerical examples are proposed to indicate the relationship of rejection penalty, machine maintenance and aging effect. Firstly, an example with 20 random jobs is proposed. Two function types of aging effects (i.e., exponential function and linear function) and 11 different levels (i.e., from 0 to 5 by the 0.5 step) are considered in the example. Based on Algorithm 1, we can obtain the optimal maintenance interval $k$, the number of rejected jobs $n_{\bar{S}}$ and the optimal total cost $T C$. According to the variation of rejection punishment, the numerical results (i.e., $k, n_{\bar{S}}$ and $T C$ ) are proposed in the following Table I, and the corresponding tendencies of $k, n_{\bar{S}}$ and $T C$ are shown in Figure 1, Figure 2 and Figure 3, respectively. 
TABLE I. The RESUlt UNDER DifFERENT REJECTED PUNISHMENT

\begin{tabular}{lllllll}
\hline \multicolumn{1}{c}{$\begin{array}{c}\text { Rejected } \\
\text { Punishment }\end{array}$} & \multicolumn{3}{c}{ Exponential aging } & \multicolumn{3}{c}{ Linear aging } \\
\cline { 2 - 7 } & $\boldsymbol{k}$ & $\mathbf{n}_{\overline{\mathbf{s}}}$ & \multicolumn{1}{c}{$\boldsymbol{T C}$} & $\boldsymbol{k}$ & $\mathbf{n}_{\overline{\mathbf{s}}}$ & \multicolumn{1}{c}{$\boldsymbol{T C}$} \\
\hline 0 & 1 & 14 & 0 & 1 & 14 & 0 \\
0.5 & 5 & 3 & 126.5 & 8 & 12 & 190.8 \\
1 & 5 & 1 & 138 & 12 & 8 & 329.4 \\
1.5 & 5 & 1 & 142 & 12 & 8 & 444.9 \\
2 & 5 & 1 & 146 & 14 & 6 & 556.45 \\
2.5 & 5 & 1 & 150 & 16 & 4 & 640.3 \\
3 & 5 & 0 & 152.3 & 16 & 4 & 709.3 \\
3.5 & 5 & 0 & 152.3 & 19 & 1 & 752.5 \\
4 & 5 & 0 & 152.3 & 19 & 1 & 766 \\
4.5 & 5 & 0 & 152.3 & 19 & 1 & 779.5 \\
5 & 5 & 0 & 152.3 & 20 & 0 & 779.75 \\
\hline
\end{tabular}

As depicted in Figure 1, Figure 2 and Figure 3, both $\mathrm{k}$ and TC are increasing and $n_{\bar{S}}$ is decreasing while the rejected punishment is increasing. In addition, almost all the values of $k, n_{\bar{S}}$ and $T C$ of the optimal schedule under the linear aging model are bigger than that of the optimal schedule under the exponential aging model.

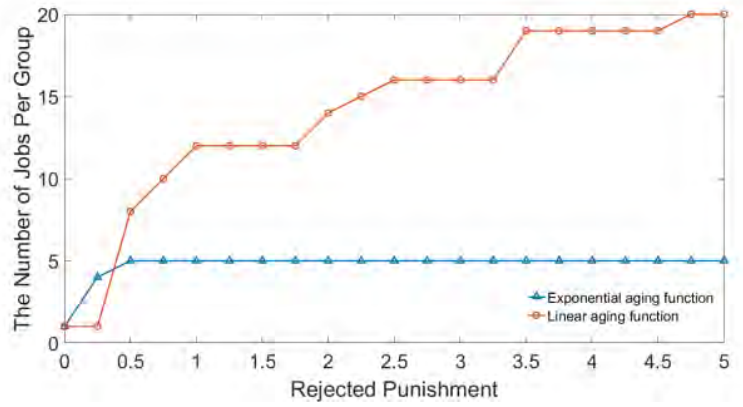

Figure 1. The number of jobs per group $k$ under different rejected punishment.

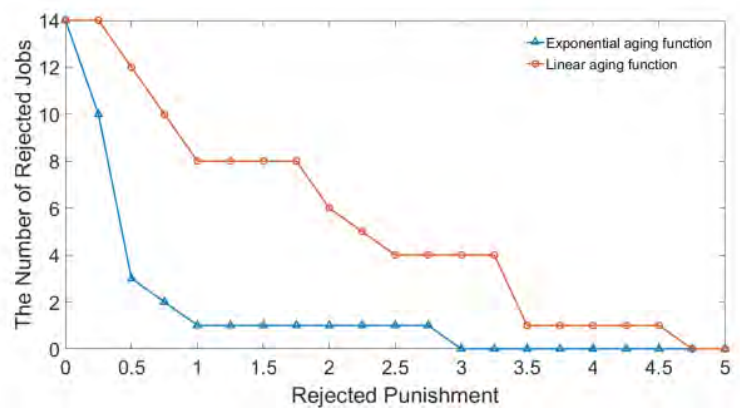

Figure 2. The number of rejected jobs $n_{\bar{S}}$ under different rejected punishment.

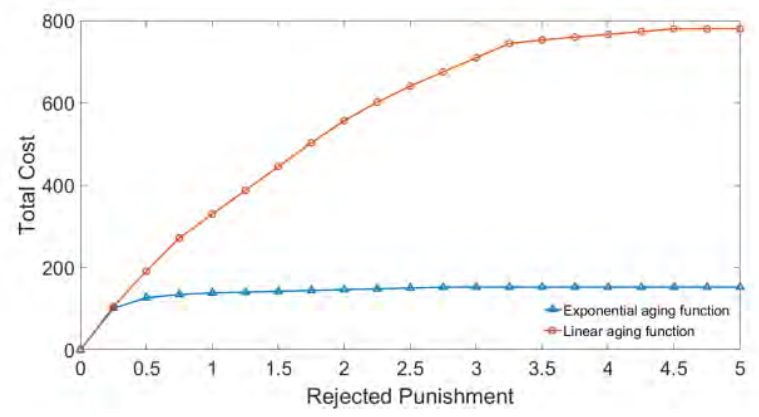

Figure 3. The total cost $T C$ under different rejected punishment.
Similarly, according to different lengths of time window, the numerical results (i.e., $k, n_{\bar{S}}$ and $T C$ ) are proposed in the following Table II, and the variation tendencies of $k, n_{\bar{S}}$ and $T C$ are depicted in Figure 4, Figure 5 and Figure 6, respectively.

TABLE II. The Result Under DifFERENT Time Window

\begin{tabular}{lllllll}
\hline \multicolumn{1}{c}{ Time } & \multicolumn{3}{c}{ Exponential aging } & \multicolumn{3}{c}{ Linear aging } \\
\cline { 2 - 7 } \multicolumn{1}{c}{ Window } & $\boldsymbol{k}$ & $\mathbf{n}_{\overline{\mathbf{s}}}$ & \multicolumn{1}{c}{$\boldsymbol{T C}$} & \multicolumn{1}{c}{$\boldsymbol{k}$} & $\mathbf{n}_{\overline{\mathbf{s}}}$ & \multicolumn{1}{c}{$\boldsymbol{T C}$} \\
\hline 0 & 1 & 0 & 0 & 1 & 0 & 0 \\
0.5 & 1 & 0 & 0 & 1 & 0 & 0 \\
1 & 1 & 0 & 0 & 1 & 0 & 0 \\
1.5 & 5 & 0 & 242 & 20 & 0 & 333.5 \\
2 & 6 & 2 & 275.5 & 1 & 19 & 612 \\
2.5 & 6 & 2 & 355.75 & 1 & 19 & 620.25 \\
3 & 7 & 6 & 403.5 & 1 & 19 & 628.5 \\
3.5 & 7 & 6 & 492.75 & 1 & 19 & 636.75 \\
4 & 8 & 4 & 528 & 1 & 19 & 645 \\
4.5 & 8 & 12 & 589.5 & 1 & 19 & 653.25 \\
5 & 9 & 11 & 606.5 & 1 & 19 & 661.5 \\
5.5 & 1 & 19 & 671.25 & 1 & 19 & 669.75 \\
6 & 1 & 19 & 679.5 & 1 & 19 & 678 \\
\hline
\end{tabular}

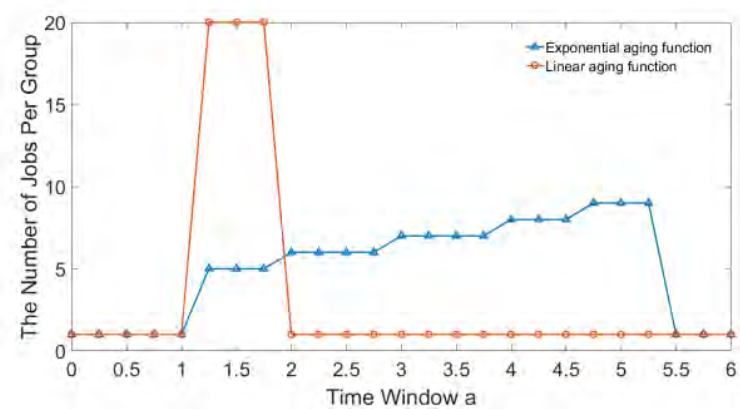

Figure 4. The number of jobs per group under different time window

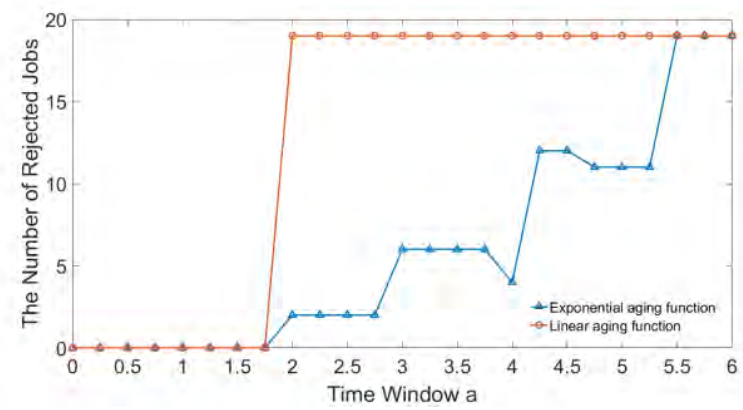

Figure 5. The number of rejected jobs under different time window.

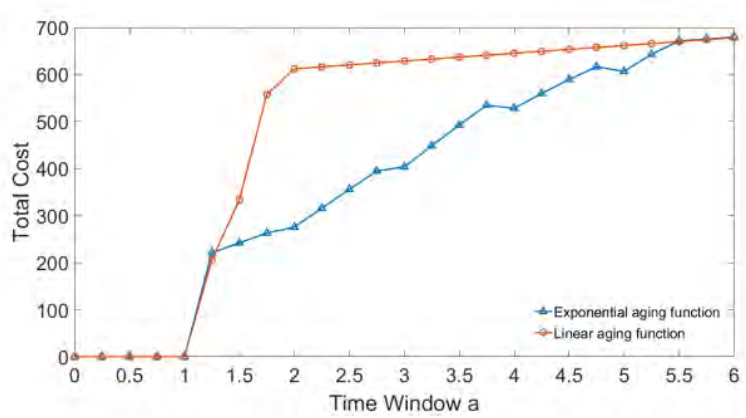

Figure 6. The total cost under different time window. 
From Figure 4, Figure 5 and Figure 6, it can be seen that $k$ is shown as an inverse "U" type, and both $T C$ and $n_{\bar{S}}$ are increasing according to the increasing length of time window. The values of $n_{\bar{S}}$ and $T C$ of the optimal schedule under the linear aging model are bigger than that of the optimal schedule under the exponential aging model.

\section{CONCLUSION}

In this paper, we studied a single machine scheduling problem with piece-rate machine maintenance; interval constrained actual processing time and rejection. To minimize the production fees, the optimal polynomial algorithm is converted to solve the considered problem, and its computational complexity is also analyzed. And then, numeric experiments are conducted to illustrate that both the total cost and the rejected job number are increasing according to the increasing length of time window, and they are decreasing where the rejected punishment is increasing.

The future work is value to analyze the problem on the other machine setting in the dynamic environment.

\section{ACKNOWLEDGEMENT}

This work is supported by the National Nature Science Foundation of China (71501098, 71603135, 71774080), China Postdoctoral Science Foundation Funded Project (164117), the College Graduate Research and Innovation Foundation of Jiangsu Province (KFJJ20170911) and the NUAA research funding (NS2016080).

\section{REFERENCES}

[1] Bartal, Y., Leonardi, S., Marchetti-Spaccamela, A., Sgall, J., and Stougie, L. (1995). Multiprocessor scheduling with rejection. None, ti 95-(1):64-78.

[2] Biskup, D. (1999). Single-machine scheduling with learning considerations. European Journal of Operational Research, 115(1):173-178.

[3] Cheng, T. C. E. and Wang, G. (2000). Single machine scheduling with learning effect considerations. Annals of Operations Research, 98(1):273-290.

[4] Cheng, Y. and Sun, S. (2009). Scheduling linear deteriorating jobs with rejection on a single machine. European Journal of Operational Research, 194(194):18-27.

[5] Engels, D. W., Karger, D. R., Kolliopoulos, S. G., Sengupta, S., Uma, R. N., and Wein, J. (1998). Techniques for scheduling with rejection. Springer Berlin Heidelberg.

[6] Gerstl, E., Mor, B., and Mosheiov, G. (2017). Minmax scheduling with acceptable lead-times: Extensions to position-dependent processing times, due-window and job rejection. Computers Operations Research, 83:150-156.

[7] Graham, R. L., Lawler, E. L., Lenstra, J. K., and Kan, A. H. G. R. (1979). Optimization and approximation in deterministic sequencing and scheduling: a survey. Annals of Discrete Mathematics, 5(1):287326.

[8] He, C., Leung, J. Y.-T., Lee, K., and Pinedo, M. L. (2016). Scheduling a single machine with parallel batching to minimize makespan and total rejection cost. Discrete Applied Mathematics, 204:150 - 163.

[9] Hsu, C. J., Yang, S. J., and Yang, D. L. (2011). Two due date assignment problems with position-dependent processing time on a single-machine. Computers Industrial Engineering, 60(4):796-800.
[10] Kuo, W. H. and Yang, D. L. (2008). Minimizing the makespan in a single-machine scheduling problem with the cyclic process of an aging effect. Journal of the Operational Research Society, 59(3):416-420.

[11] Li, G., Liu, M., Sethi, S. P., and Xu, D. (2017). Parallel-machine scheduling with machine-dependent maintenance periodic recycles. International Journal of Production Economics, 186:1 - 7.

[12] Liao, C. and Chen, W. (2003). Single-machine scheduling with periodic maintenance and nonresumable jobs. Computers Operations Research, 30(9):1335 - 1347.

[13] Luo, W. and Liu, F. (2017). On single-machine scheduling with workload-dependent maintenance duration. Omega, 68:119 - 122.

[14] Mor, B. and Mosheiov, G. (2012). Scheduling a maintenance activity and due-window assignment based on common flow allowance. International Journal of Production Economics, 135(1):222-230.

[15] Mosheiov, G. (2001). Parallel machine scheduling with a learning effect. Journal of the Operational Research Society, 52(10):11651169.

[16] Mosheiov, G. and Oron, D. (2012). Minimizing the number of tardy jobs on a proportionate flowshop with general position-dependent processing times. Computers Operations Research, 39(7):1601-1604.

[17] Mosheiov, G. and Sidney, J. B. (2003). Scheduling with general job-dependent learning curves. European Journal of Operational Research, 147(3):665-670.

[18] Sengupta, S. (2003). Algorithms and approximation schemes for minimum lateness/tardiness scheduling with rejection. Lecture Notes in Computer Science, 2748:79-90.

[19] Wang, J. B. and Wang, M. Z. (2011). Worst-case behavior of simple sequencing rules in flowshop scheduling with general position-dependent learning effects. Annals of Operations Research, 191(1):155-169.

[20] Xu, D., Sun, K., and Li, H. (2008). Parallel machine scheduling with almost periodic maintenance and non-preemptive jobs to minimize makespan. Computers \& Operations Research, 35(4):1344 - 1349.

[21] Xue, P., Zhang, Y., and Yu, X. (2014). Single-machine scheduling with piece-rate maintenance and interval constrained position-dependent processing times. Applied Mathematics Computation, 226(1):415-422.

[22] Yang, D. L., Cheng, T. C. E., Yang, S. J., and Hsu, C. J. (2012). Unrelated parallel-machine scheduling with aging effects and multi-maintenance activities. Computers Operations Research, 39(7):1458-1464.

[23] Yang, S. J. and Yang, D. L. (2010). Minimizing the makespan on single-machine scheduling with aging effect and variable maintenance activities. Omega, 38(6):528-533.

[24] Yin, Yunqiang, Cheng, T., C. E., Xu, Jianyou, Cheng, Shuenn-Ren, Wu, and Chin-Chia (2013). Single-machine scheduling with past-sequence-dependent delivery times and a linear deterioration. Journal of Industrial Management Optimization, 9(2):323-339.

[25] Yu, X. and Zhang, Y. (2014). Single machine scheduling with aging effect and upper-bounded actual processing times. Arabian Journal for Science and Engineering, 39(2):1489-1495.

[26] Yu, X., Zhang, Y., Xu, D., and Yin, Y. (2013). Single machine scheduling problem with two synergetic agents and piece-rate maintenance. Applied Mathematical Modelling, 37(3):1390-1399.

[27] Zhang, L., Lu, L., and Yuan, J. (2009). Single machine scheduling with release dates and rejection. European Journal of Operational Research, 36(10):2748-2751.

[28] Zhao, C. L. and Tang, H. Y. (2010). Single machine scheduling with general job-dependent aging effect and maintenance activities to minimize makespan. Applied Mathematical Modelling, 34(3):837-841.

[29] Zou, J. and Zhang, Y. (2014). Scheduling simple linear deteriorating jobs with rejection. Mathematical Problems in Engineering, 2014(3):1-7. 